\title{
CpG hypermethylation of human four-and-a-half LIM domains 1 contributes to migration and invasion activity of human bladder cancer
}

\author{
MITSUGI MATSUMOTO ${ }^{1}$, KAZUMORI KAWAKAMI ${ }^{1}$, HIDEKI ENOKIDA ${ }^{1}$, KAZUKI TOKI ${ }^{1}$, \\ RYOICHIRO MATSUDA ${ }^{1}$, TAKESHI CHIYOMARU ${ }^{1}$, KENRYU NISHIYAMA ${ }^{1}$, \\ KAZUYA KAWAHARA ${ }^{2}$, NAOHIKO SEKI ${ }^{3}$ and MASAYUKI NAKAGAWA ${ }^{1}$ \\ ${ }^{1}$ Department of Urology, Graduate School of Medical and Dental Sciences, Kagoshima University; \\ ${ }^{2}$ Kawahara Nephro-urology Clinic, Kagoshima; ${ }^{3}$ Department of Functional Genomics, \\ Graduate School of Medicine, Chiba University, Chiba, Japan
}

Received December 16, 2009; Accepted January 25, 2010

DOI: $10.3892 /$ ijmm_00000458

\begin{abstract}
We previously reported a simple technique that combines microarray data from clinical bladder cancer (BC) specimens with those from a BC cell line (BOY) treated with a pharmacologic demethylating agent (5-aza-dC). We focused on the human four-and-a-half LIM domains 1 (FHL1) gene which was selected on the basis of previous microarray data analysis. Because LIM domains provide protein-protein binding interfaces, FHL genes play an important role in cellular events, such as focal adhesion and differentiation, by interacting with the target protein as either a repressor or activator. We hypothesized that inactivation of the FHL1 gene through $\mathrm{CpG}$ methylation contributes to cell viability including migration and invasion activity of human BC. After 5-aza-dC treatment, the expression levels of FHL1 mRNA transcript markedly increased in all cell lines tested, as shown by real-time reverse transcription-polymerase chain reaction (RT-PCR). The methylation index of FHL1 in our samples was significantly higher in $70 \mathrm{BC}$ specimens than in 10 normal bladder epithelium (NBE) specimens $(63.9 \pm 25.5$ and $0.3 \pm 0.2$, respectively; p=0.0066). Conversely, FHL1 mRNA expression was significantly lower in the BC specimens than in the NBE ones $(0.331 \pm 0.12$ and $2.498 \pm 0.61$, respectively; $\mathrm{p}=0.0011)$. In addition, significant inhibitions of wound healing $(45.78 \pm 6.2$, and $100 \pm 0$, respectively; $\mathrm{p}=0.009)$ and of cell invasion (18.5 \pm 2.3 and $95.2 \pm 2.4$, respectively; $\mathrm{p}=0.02$ ) were observed in stable $F H L 1$-transfected cells than in the control BC cells. In conclusion, we found that the
\end{abstract}

Correspondence to: Dr Kazumori Kawakami, Department of Urology, Graduate School of Medical and Dental Sciences, Kagoshima University, 8-35-1 Sakuragaoka, Kagoshima 890-8520, Japan

E-mail:kkawakam@m.kufm.kagoshima-u.ac.jp

Key words: bladder cancer, microarray, FHL1, methylation mechanism of FHL1 down-regulation in $\mathrm{BC}$ is through $\mathrm{CpG}$ hypermethylation of the promoter region. FHL1 gene inactivation by $\mathrm{CpG}$ hypermethylation may thus contribute to migration and invasion activity of $\mathrm{BC}$.

\section{Introduction}

Bladder cancer (BC), one of the five most common malignancies in the US, is the second most common tumor of the genitourinary tract and the second most common cause of death in patients with urinary tract malignancies (1). As in other human cancers, loss of function of tumor suppressor genes and activation of oncogenes are two major alterations that promote $\mathrm{BC}$ initiation and progression (2). Our laboratory developed a simple technique that combines microarray data from clinical $\mathrm{BC}$ specimens with those from a $\mathrm{BC}$ cell line (BOY) treated with a pharmacologic demethylating agent $(5$-aza-dC) $(3,4)$. As described in a previous report $(4)$, we used it to identify the methylated gene profile in human $\mathrm{BC}$; this profile contains 24 methylated genes. Among these genes, we focused on the human four-and-a-half LIM domains 1 (FHL1) gene, one of the down-regulated genes in the gene expression profile of human BC (3).

The FHL family genes are characterized by LIM domains. Because LIM domains provide protein-protein binding interfaces, $F H L$ genes play an important role in cellular events, such as focal adhesion and differentiation, by interacting with the target protein as either a repressor or activator (5-7). The molecules associated with intercellular adhesion are possibly inhibited during tumor progression (8). The expression of the FHL1 gene, located at human chromosome Xq26, is downregulated in various types of malignancies including lung, prostate, breast, ovarian, colon, and thyroid cancer, brain tumors, renal cancer, liver cancer, melanoma, and gastric cancer (9-13).

Gene function can be altered by epigenetic mechanisms. Silencing of tumor suppressor genes by hypermethylation of $\mathrm{CpG}$ islands within the promoter and/or 5'-regions is a common feature of human cancer and is often associated 
with partial or complete transcriptional blocking. Many tumor suppressor genes silenced by DNA methylation have been identified in various types of cancer including BC $(14,15)$. However, little is known about the status of FHLI promoter methylation and the functional role of FHLl in BC. We hypothesized that inactivation of the FHL1 gene through $\mathrm{CpG}$ methylation contributes to cell viability including migration and invasion activity of human BC. To test this hypothesis, we investigated the mRNA expression levels in $\mathrm{BC}$ cell lines and human BC tissue. Methylation status was determined by real-time quantitative methylation-specific polymerase chain reaction (PCR) in BC specimens. In addition, we established stable FHL1-transfected cells and compared their proliferation, migration, and invasion activities with those of the control $\mathrm{BC}$ cell line.

\section{Materials and methods}

Clinical samples. A total of 70 pathologically proven $\mathrm{BC}$ specimens were obtained from BC patients who underwent TUR-Bt or radical cystectomy at Kagoshima University Hospital and affiliated hospitals between 2003 and 2008. Also obtained were ten normal bladder epithelium (NBE) specimens from patients without BC. The background and clinicopathological characteristics of the patients are summarized in Table I. Each tumor was staged and graded in accordance with the tumor-node-metastasis (TNM) staging system (16). The study was approved by the Institutional Review Board of our institution; written prior informed consent was obtained from all patients for use of their specimens and clinical and pathological data.

Cell culture and 5-Aza-dC treatment. The human BC cell lines (T24, UMUC and BOY) were maintained in MEM mixed with $10 \% \mathrm{FBS}$ and $1 \%$ penicillin/streptomycin in a humidified atmosphere of $5 \% \mathrm{CO}_{2}$ and $95 \%$ air at $37^{\circ} \mathrm{C}$. To screen for epigenetic alterations of gene methylation, these cell lines were treated with a DNA methyltransferase inhibitor, 5-aza-dC (Sigma-Aldrich, St. Louis, MO). Cultured cells were harvested after seven days of exposure to $10 \mu \mathrm{M}$ of 5-aza-dC, and total RNA was extracted from the cell lines before and after drug treatment.

Nucleic acid extraction. Genomic DNA was extracted from the BC and NBE specimens with a QIAamp tissue kit (Qiagen, Valencia, CA) after microdissection of $10-\mu$ m-thick paraffinembedded sections. It was precipitated with ethanol. Total RNA was extracted from the clinical specimens using Isogen (Nippon Gene, Tokyo, Japan), following the manufacturer's protocol. Total RNA was extracted from the human BC cell lines with a QIAamp DNA/RNA Mini kit (Qiagen) according to the manufacturer's instructions. The concentration of nucleic acid was determined with a spectrophotometer, and the integrity was checked by gel electrophoresis.

cDNA preparation and real-time quantitative RT-PCR. First strand cDNA with $1 \mu \mathrm{g}$ of total RNA was synthesized using random primers of high capacity cDNA reverse transcription kit (Applied Biosystems). Premium total RNA from normal human bladder (Clontech, Palo Alto, CA) was used as a normal
Table I. Patient characteristics.

\begin{tabular}{|c|c|c|}
\hline \multicolumn{3}{|l|}{ Bladder cancer $(\mathrm{BC})$} \\
\hline Total number & 70 & \\
\hline Median age (range) & 74 & $(49-100)$ yrs \\
\hline \multicolumn{3}{|l|}{ Gender } \\
\hline Male & 46 & \\
\hline Female & 24 & \\
\hline \multicolumn{3}{|l|}{ Stage } \\
\hline Tis & 1 & \\
\hline Superficial (pTa) & 16 & \\
\hline Invasive ( $\geq \mathrm{pT} 1)$ & 53 & \\
\hline \multicolumn{3}{|l|}{ Grade } \\
\hline G1 & 5 & \\
\hline $\mathrm{G} 2$ & 40 & \\
\hline G3 & 22 & \\
\hline Unknown & 3 & \\
\hline \multicolumn{3}{|l|}{ Operation } \\
\hline Cystectomy & 8 & \\
\hline TUR-Bt & 62 & \\
\hline \multicolumn{3}{|l|}{ Recurrence } \\
\hline Recurrence & 36 & \\
\hline Recurrence (-) & 29 & \\
\hline Unknown & 5 & \\
\hline Follow-up period (range) & 513 & (13-1440) days \\
\hline \multicolumn{3}{|c|}{ Normal bladder epithelium (NBE) } \\
\hline Total number & 10 & \\
\hline Median age (range) & 66 & $(58-76)$ yrs \\
\hline \multicolumn{3}{|l|}{ Gender } \\
\hline Male & 8 & \\
\hline Female & 2 & \\
\hline
\end{tabular}

bladder control. Gene-specific PCR products were continuously measured using an Applied Biosystems 7300 real-time PCR system, following the manufacturer's protocol. The first PCR step was a 10 min hold at $95^{\circ} \mathrm{C}$ followed by 45 cycles and the 45 cycles of a $15 \mathrm{sec}$ denaturation step at $95^{\circ} \mathrm{C}$ and a $1 \mathrm{~min}$ annealing/extension step at $60^{\circ} \mathrm{C}$. All reactions were performed in triplicate, and a negative control lacking cDNA was included. TaqMan ${ }^{\circledR}$ probes and primers for $F H L 1(\mathrm{P} / \mathrm{N}$ : Hs00793641_g1, Applied Biosystems) and Glucuronidase $\beta$ (GUSB) (P/N: Hs99999908_m1, Applied Biosystems) were the assay-on-demand gene expression products. The expression of FHL1 mRNA was normalized to the amount of GUSB in the same cDNA using the standard curve method provided by the manufacturer.

Real-time quantitative methylation-specific PCR. For TaqMan quantitative methylation-specific PCR (QMSP), the fluorescent probe and primer sets were designed to hybridize to the amplified region of DNA (Fig. 1). Myogenic differentiation-1 (MYOD1) primer sequences were used as an internal control (4). The first PCR was amplified with universal primers (Pan-S; GGtTtgtTattaAGGGGAGG and Pan-As; 


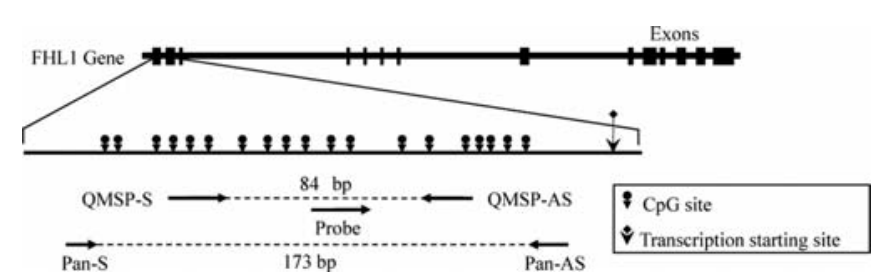

Figure 1. Schematic representation of location of $\mathrm{CpG}$ sites and primers designed within functional promoter of FHL1 gene. First, universal PCR was performed with universal primers Pan-sense (S) and Pan-antisense (AS), which do not carry any CpG sites. Quantitative MSP was done using primer sets MSP-S and MSP-AS, and USP-S and USP-AS, respectively, and the universal PCR product was used as a template. For TaqMan QMSP, the fluorescent probe and primer sets were designed to hybridize to the amplified DNA region.

ACAAAAACAACCAAATAAAAATAAC) using $3 \mu \mathrm{l}$ of bisulfite-treated DNA in a total volume of $20 \mu 1$. The first PCR step was 10 min hold at $95^{\circ} \mathrm{C}$ followed by 45 cycles of a 1 min denaturation step at $95^{\circ} \mathrm{C}$, a 1 min annealing step at $48^{\circ} \mathrm{C}$, and a 1 -min extension step at $72^{\circ} \mathrm{C}$. A second round of nested QMSP was then carried out using $1 \mu 1$ of the first PCR product with the same PCR conditions described above except for the annealing temperature $\left(59^{\circ} \mathrm{C}\right)$. The PCR cycle was programmed up to 45 cycles, and all reactions were performed in duplicate. The primers and probe sequences were QMSP primers, 5'-GTCGTTGTAAGTTATCGGGTTTC-3' (sense), 5'-CTACGCACTCCCTAAAAACG-3' (antisense), and 5'-A GGTCGAAACGGCGTGG-3' (probe). Serial dilutions of CpGenome universal methylated DNA (Millipore, Tokyo, Japan) were used to construct a calibration curve. The methylation index was defined as the quantity of fluorescence intensity derived from $F H L 1$ promoter amplification divided by the fluorescence intensity from MYOD1 amplification, multiplied by 1000 .

Immunoblotting. Total protein lysate was prepared with detergent lysis buffer in the presence of a protease inhibitor. Twenty micrograms of protein lysate was separated by NuPAGE on 4-12\% bis-tris gel (Invitrogen, Tokyo, Japan) and transferred into a PVDF membrane. Immunoblotting was done with diluted (1/200) polyclonal FHL1 antibody (109911-AP, Protein Tech Group, Chicago, IL). After being washed, the membrane was incubated with goat anti-rabbit IgG horseradish peroxidase conjugate (Bio-Rad, Hercules, CA). Specific complexes were visualized with an echochemiluminescence detection system (GE Healthcare Bio-Sciences, Buckinghamshire, UK).

Construction of FHL1 expression vectors and transfection to BOY cells. The FHL1 vector was constructed by inserting full-length FHL1 cDNA into the Sse83871 and HindIII restriction site of the pBApo-CMV Neo ${ }^{\mathrm{TM}}$ vector (Takara Bio, Otsu, Japan). The FHL1 and non-targeting (control) vector were transfected into BOY cells by calcium phosphate co-precipitation. The cells were then split and grown in selective medium with $1000 \mu \mathrm{g} / \mathrm{ml}$ of G418. After 2 weeks, G418-resistant colonies were chosen and expanded in medium containing $100 \mu \mathrm{g} / \mathrm{ml}$ of $\mathrm{G} 418$.

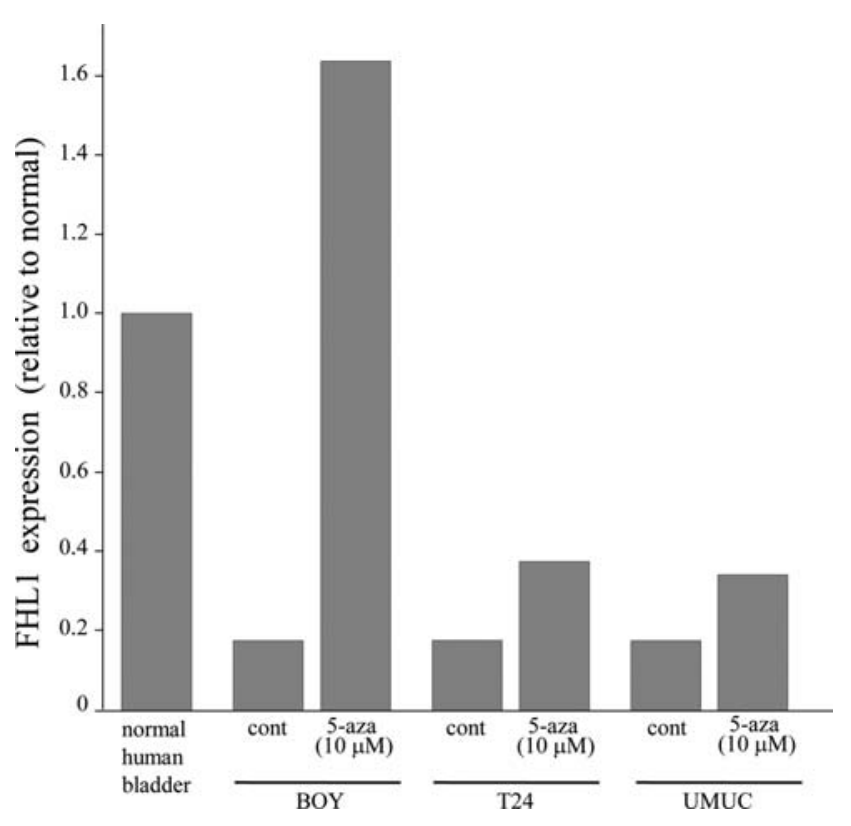

Figure 2. FHL1 mRNA expression in BC cells before and after treatment with 5-aza-dC. Quantitative real-time RT-PCR demonstrated that mRNA transcript of FHL1 gene was markedly increased after 5-aza-dC treatment especially in BOY cells. PCR conditions and primer sequences are described in Materials and methods section.

XTT assays. FHL1-transfected, control, and wild-type BOY cells were transferred to 96 -well plates $\left(3 \times 10^{3}\right.$ cells/well $)$ and incubated for $72 \mathrm{~h}$. A $50 \mu 1$ solution of XTT $(0.3 \mathrm{mg} / \mathrm{ml})$ containing $1.25 \mu \mathrm{M}$ of PMS was then added to each well. The absorbance at $492 \mathrm{~nm}$ was measured after another $4 \mathrm{~h}$ of incubation at $37^{\circ} \mathrm{C}$.

Wound healing assay. FHL1-transfected, control, and wild-type BOY cells were initially seeded uniformly onto $60 \mathrm{~mm}$ culture plates. An artificial 'wound' was carefully created at $0 \mathrm{~h}$ by using a P-20 pipette tip to scratch the subconfluent cell monolayer. Microphotographs were taken at 0 and $12 \mathrm{~h}$. Quantitative analysis of the percentage of wound healing was calculated using the distance across the wound $(n=5)$ at 0 and $12 \mathrm{~h}$, divided by the distance measured at $0 \mathrm{~h}$ for each cell line. The experiment was repeated twice in sets of two, independently.

Invasion assay. A cell invasion assay was carried out using modified Boyden chambers consisting of Transwell-precoated Matrigel membrane filter inserts with $8 \mu \mathrm{m}$ pores in 24 -well tissue culture plates (BD Biosciences, Bedford, MA). Two days after transfection with $F L H-1,5 \times 10^{4}$ BOY cells were placed in the top of the chamber in serum-free DMEM, and the bottom chamber was filled with DMEM containing $10 \%$ FBS as a chemoattractant. After $22 \mathrm{~h}$ of incubation in $5 \%$ $\mathrm{CO}_{2}$ humidity at $37^{\circ} \mathrm{C}$, non-invading cells were removed from the upper filter surfaces, and the filters were washed, fixed, and stained using a Diff-Quick kit (Sysmex, Kobe, Japan). Four randomly selected fields, magnification x200, were photographed, and the number of invading cells in each was counted. The experiment was repeated twice in sets of two, independently. 

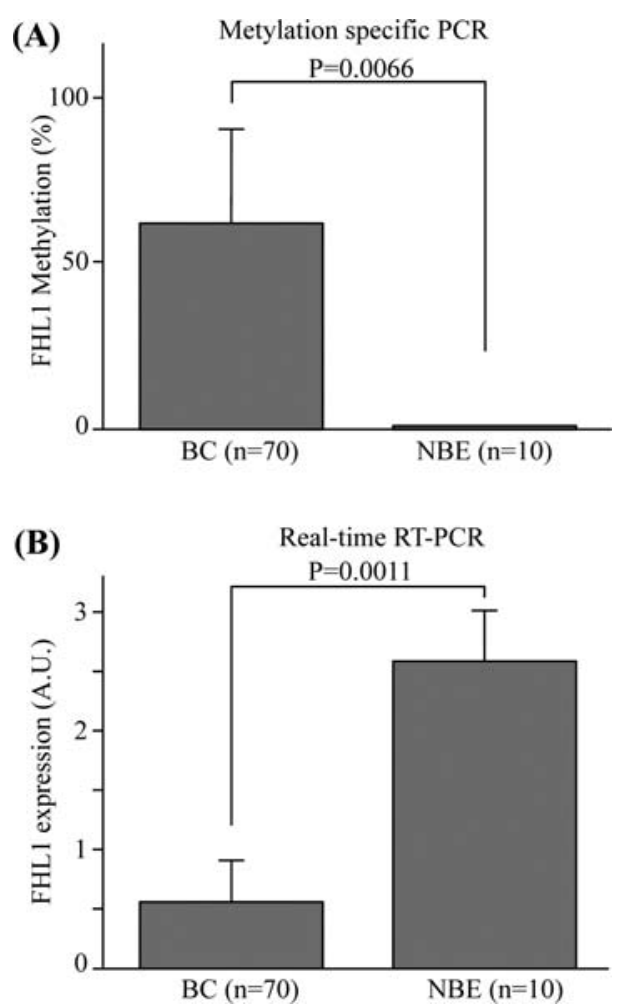

Figure 3. FHL1 methylation index and mRNA expression of BC and NBE specimens. (A) FHL1 methylation index was significantly higher for BC $(\mathrm{p}=0.0066)$. Methylation index was defined as described in Materials and methods section. (B) FHL1 mRNA expression was significantly lower in BC $(\mathrm{p}=0.0011)$.

Statistical analysis. The relationship between two groups and the numerical value obtained by real-time RT-PCR was analyzed using the Mann-Whitney $U$ test. The relationship between the BC and NBE specimens with FHL1 methylation status was carried out using the $\chi^{2}$ test. The analysis software was Expert StatView (version 4, SAS Institute Inc., Cary, NC), and the differences of $\mathrm{p}<0.05$ were considered statistically significant.

\section{Results}

Validation of altered FHL1 mRNA expression in BC cell lines before and after 5-aza-dC treatment. The three BC cell lines (T24, UMUC and BOY) were subjected to real-time RT-PCR experiments to evaluate the mRNA expression levels of FHL1 (Fig. 2). Before 5-aza-dC treatment, the FHL1 mRNA expression levels in the BC cell lines were downregulated and were lower than those of the normal human bladder. After 5-aza-dC treatment, the FHL1 mRNA expression was up-regulated in the $\mathrm{BC}$ cell lines, and it was markedly higher in the BOY cells, which had been subjected to cDNA microarray analysis for obtaining the methylated gene profile in BC. Hence, the pattern of FHL1 mRNA expression by 5 -aza-dC treatment was validated by both real-time RT-PCR and cDNA microarray analysis (4).

Methylation status of FHL1 gene promoter and FHL1 mRNA expression in human BC specimens. We used QMSP and realtime quantitative RT-PCR to evaluate the methylation status of the FHL1 promoter region and the expression levels of FHL1 mRNA. The methylation index of the BC specimens was significantly higher than that of the NBE ones $(63.9 \pm 25.5$ and $0.3 \pm 0.2$, respectively; $\mathrm{p}=0.0066$ ) (Fig. $3 \mathrm{~A}$ ). In contrast, the expression level of FHL1 mRNA in the BC specimens was significantly lower than that in the NBE ones $(0.331 \pm 0.12$ and 2.498 \pm 0.61 , respectively; $\mathrm{p}=0.0008$ ) (Fig. 3B). These results indicate that FHL1 $m R N A$ expression might be downregulated by methylation of the FHLl promoter region. We found no relationship between the clinicopathological parameters and the FHL1 methylation index or mRNA expression (data not shown).
(A)

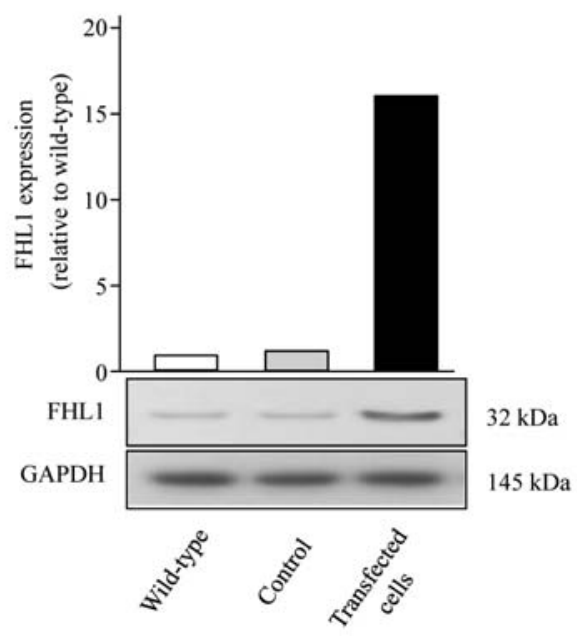

(B)

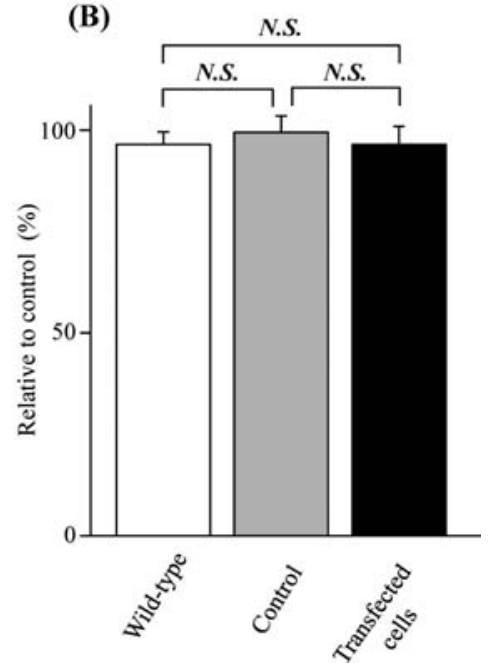

Figure 4. Effect of FHL1 transfection on cell growth. (A) Expression level of FHL1 mRNA was more than 16-fold higher in FHL1-transfected cells than in the control and the wild-type cells (upper; real-time RT-PCR). Western blot analysis revealed high expression levels of FHL1 in FHL1-transfected cells compared to control and wild-type cells (lower). (B) Cell growth determined by XTT assay. There was no significant difference in anchorage-dependent cell growth for FHL1-transfected, control, and wild-type BOY cells. 
(A)
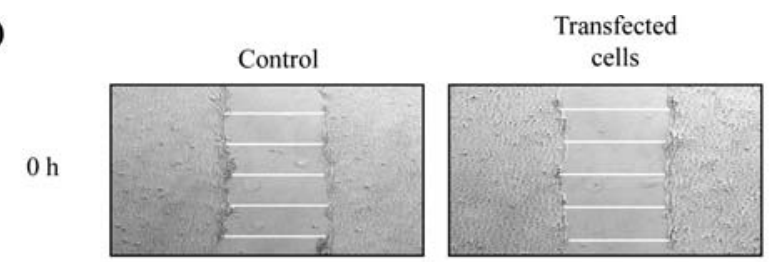

$12 \mathrm{~h}$
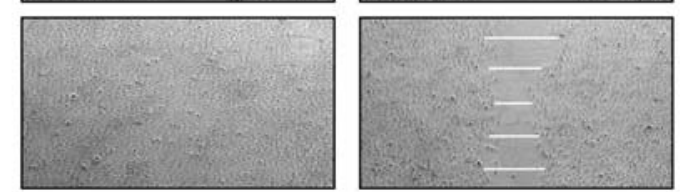

(B)

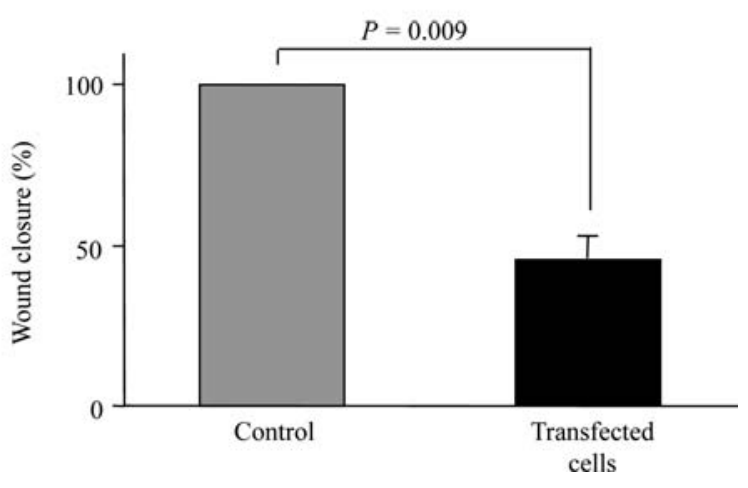

Figure 5. Effect of FHL1 transfection on cell migration. (A) Representative photographs of cell migration activity from the wound healing assay. Wound was completely closed at $12 \mathrm{~h}$ after scratching in control cells whereas it was still open in FHL1-transfected cells. (B) Percentage of wound closure corres-ponding to distance between wound edges at $12 \mathrm{~h}$ in five randomly chosen regions (mean $\pm \mathrm{SD}$ ) relative to distance at time $0 \mathrm{~h}$ for each cell.
Identification of FHL1 expression in transfected cells and effect of FHL1 overexpression on cell proliferation. Because we found an extremely low expression of FHL1 mRNA and a marked retrieval of the gene expression after 5-aza-dC treatment in the BOY cells (Fig. 2), we established stable FHL1-transfected BOY cells. The expression level of FHL1 mRNA was more than 16-fold higher in the transfected cells than in the control and wild-type cells (Fig. 4A, upper). Western blot analysis demonstrated that the expression levels of the FHL1-transfected cells were high (Fig. 4A, lower). For gain-of-function experiments, we first performed XTT cell growth assay using FHL1-transfected, control, and wild-type BOY cells. We found no significant difference in anchoragedependent cell growth among the three groups from three independent assays (Fig. 4B).

Effect of FHL1 transfection on cellular migration and invasion. To evaluate cellular migration, we performed wound healing assay using FHL1-transfected and control BOY cells. As shown in Fig. 5A, the cell migration ability of the FLH1transfected cells was less than that of the control cells. Two independent wound healing assays showed significant wound healing inhibition after 12-h incubation in the FHL-1 transfected cells compared to the control cells $(45.78 \pm 6.2$ and $100 \pm 0$, respectively; p<0.009) (Fig. 5B). Next, we evaluated the invasive capacity of the $\mathrm{BC}$ cell lines using an in vitro invasion assay since penetration of extracellular matrix and basement membrane by cancer cells is a key step in tumor invasion. As shown in Fig. 6A, there were fewer invading cells in the FHL1-transfected cells than in the control cells. The number of invading cells was significantly lower in the FHL1-transfected cells than in the control cells $(18.5 \pm 2.3$ and 95.2 \pm 2.4 , respectively; $\mathrm{p}=0.02)$ (Fig. 6B).
(A)

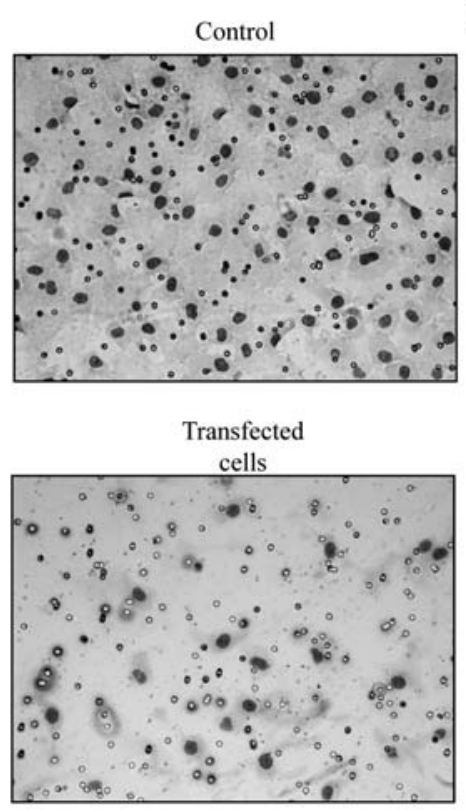

(B)

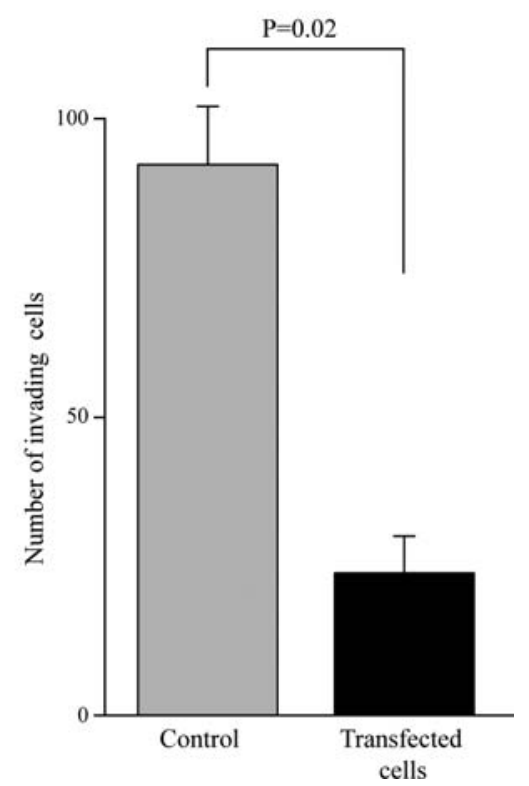

Figure 6. Effect of FHL1 transfection on cell invasion. (A) Representative photographs of cell invasion activity from the Matrigel invasion assay. Number of invading cells was lower in FHL1-transfected cells than in control cells. (B) Number of invading cells counted in four random fields (mean \pm SD). FHL1transfected cells had significantly fewer invading cells. 


\section{Discussion}

The four-and-a-half LIM (FHL) proteins are characterized by four complete LIM domains preceded by an N-terminal half LIM domain. LIM domains are cysteine-rich zinc finger motifs involved in a wide range of protein-protein interactions (17). Through interaction with cellular proteins, FHL proteins regulate cellular processes, including proliferation, differentiation, apoptosis, adhesion, migration, transcription, and signal transduction (18-22). Among these FHL family genes, we focused on FHL1, which was selected on the basis of two types of microarray analysis $(3,4)$. Although the down-regulation of FHLI has been found in several types of cancer (9-13), the expression level and molecular function of FHL1 in BC have not been clarified. Since many tumor suppressor genes silenced by DNA methylation have been identified in primary BC samples $(14,15)$, we hypothesized that FHL1 expression is down-regulated by promoter hypermethylation in BC cells and that FHLl functions as a tumor suppressor in $\mathrm{BC}$ cells.

In this study, we found that FHL1 was significantly downregulated at the mRNA level in the human $\mathrm{BC}$ tissue samples and that the methylation index was significantly higher in BC specimens than in NBE ones. Treatment with demethylation agent (5-aza-dC) restored FHL1 mRNA expression in the BC cell lines. These results suggest that the down-regulation of FHL1 expression in BC cell lines and human BC tissue correlates with $\mathrm{CpG}$ hypermethylation of the $\mathrm{FHL1}$ promoter. This is in accordance with our initial hypothesis that the FHLI promoter region is hypermethylated in BC. Since there was no apparent relationship between the clinicopathological parameters and the FHL1 methylation index or mRNA expression, promoter methylation and down-regulation of FHL1 might be involved in the initial stage of tumorigenesis (23).

We also hypothesized that FHL1 functions as a tumor suppressor in BC. To examine the function of the FHLI gene, we established stable FHL1-transfected cells and compared their proliferation, migration, and invasion activities with those of the control BOY cell line, which was transfected with an empty vector. Cell proliferation assay revealed no difference in cell growth ability between the FHL1-transfected cells and the control cells. Shen et al demonstrated that FHL1-transfected cells have lower activity of non-anchored cell growth but not of anchored cell growth (9). Although the difference in the cell growth mechanism between anchored and non-anchored cells is unknown, our finding is similar to theirs. Although FHL1 restoration resulted in no significant difference on anchored cell growth, we demonstrated that it significantly inhibited cell migration and invasion. These results strongly indicate that $F H L 1$ functions as a tumor suppressor in BC cells. Ding et al demonstrated that transfection of hepatocellular cancer cell line HepG2 with FHL1 inhibited cell growth and decreased anchorageindependent colony formation whereas FHLI knockdown by siRNA increased cell growth and colony formation (17). They also found that FHL proteins ultimately increased the expression of growth inhibitor genes, such as the CDK inhibitor, $p 21$, and that they decreased the expression of the growth-promoting gene, $c-m y c$ (17). In addition, FHLI interacts with estrogen receptors and inhibits breast cancer cell growth $(24,25)$. Moreover, estrogen receptors are highly expressed in $\mathrm{BC}$ and contribute to $\mathrm{BC}$ cell proliferation $(26,27)$. Thus, previous and current findings lead us to think that FHL1 might have a tumor suppressive function through interaction with p21, c-myc, and estrogen receptors in BC. Further study is necessary to test this hypothesis.

In conclusion, we have shown that FHL1 is frequently down-regulated in BC cell lines and clinical BC tissue. The mechanism of FHL1 down-regulation in $\mathrm{BC}$ is through $\mathrm{CpG}$ hypermethylation of the promoter region. In addition, FHL1 restoration causes suppression of $\mathrm{BC}$ cell migration and invasion. These results suggest that FHL1 functions as a tumor suppressor gene in $\mathrm{BC}$ cells and that restoration of FHL1 might offer a new therapeutic approach for the treatment of $\mathrm{BC}$ patients.

\section{Acknowledgements}

We thank Ms. M. Miyazaki for her excellent laboratory assistance.

\section{References}

1. Jemal A, Siegel R, Ward E, et al: Cancer statistics, 2008. CA Cancer J Clin 58: 71-96, 2008.

2. Mitra AP, Datar RH and Cote RJ: Molecular pathways in invasive bladder cancer: new insights into mechanisms, progression, and target identification. J Clin Oncol 24: 5552$5564,2006$.

3. Kawakami K, Enokida H, Tachiwada T, et al: Identification of differentially expressed genes in human bladder cancer through genome-wide gene expression profiling. Oncol Rep 16: 521-531, 2006.

4. Mori K, Enokida H, Kagara I, et al: CpG hypermethylation of collagen type I alpha 2 contributes to proliferation and migration activity of human bladder cancer. Int J Oncol 34: 1593-1602, 2009.

5. Johannessen M, Moller S, Hansen T, et al: The multifunctional roles of the four-and-a-half-LIM only protein FHL2. Cell Mol Life Sci 63: 268-284, 2006.

6. Morgan MJ, Madgwick AJ, Charleston B, et al: The developmental regulation of a novel muscle LIM-protein. Biochem Biophys Res Commun 212: 840-846, 1995.

7. Brown S, McGrath MJ, Ooms LM, et al: Characterization of two isoforms of the skeletal muscle LIM protein 1, SLIM1. J Biol Chem 274: 27083-27089, 1999.

8. Oku N, Sasabe E, Ueta E, et al: Tight junction protein claudin-1 enhances the invasive activity of oral squamous cell carcinoma cells by promoting cleavage of laminin- $5 \mathrm{c} 2$ chain via matrix metalloproteinase (MMP)-2 and membrane-type MMP-1. Cancer Res 66: 5251-5257, 2006

9. Shen Y, Jia Z, Nagele RG, et al: Src uses Cas to suppress Fhl1 in order to promote nonanchored growth and migration of tumor cells. Cancer Res 66: 1543-1552, 2006.

10. Bhattacharjee A, Richards WG, Staunton J, et al: Classification of human lung carcinomas by mRNA expression profiling reveals distinct adenocarcinoma subclasses. Proc Natl Acad Sci USA 98: 13790-13795, 2001.

11. Fryknäs M, Wickenberg-Bolin U, Göransson H, et al: Molecular markers for discrimination of benign and malignant follicular thyroid tumors. Tumor Biol 27: 211-220, 2006.

12. Sakashita K, Mimori K, Tanaka F, et al: Clinical significance of loss of Fhl1 expression in human gastric cancer. Ann Surg Oncol 15: 2293-2300, 2008.

13. Li X, Jia Z, Shen Y, Ichikawa H, Jarvik J, Nagele RG and Goldberg GS: Coordinate suppression of Sdpr and Fhl1 expression in tumors of the breast, kidney, and prostate. Cancer Sci 99: 1326-1333, 2008.

14. Enokida $\mathrm{H}$ and Nakagawa M: Epigenetics in bladder cancer. Int J Clin Oncol 13: 298-307, 2008. 
15. Lin HH, Ke HL, Huang SP, et al: Increase sensitivity in detecting superficial, low grade bladder cancer by combination analysis of hypermethylation of E-cadherin, p16, p14, RASSF1A genes in urine. Urol Oncol (In press).

16. Sobin LH and Wittekind C: TNM Classification of Malignant Tumours, 6th edition. International Union Against Cancer (UICC). Wiley-Liss Publications, New York, 2002.

17. Ding L, Wang Z, Yan J, et al: Human four-and-a-half LIM family members suppress tumor cell growth through a TGF-B-like signaling pathway. J Clin Invest 119: 349-361, 2009.

18. Samson T, Smyth N, Janetzky S, et al: The LIM-only proteins FHL2 and FHL3 interact with alpha- and beta-subunits of the muscle alpha7beta1 integrin receptor. J Biol Chem 279: 28641-28652, 2004.

19. Coghill ID, Brown S, Cottle DL, et al: FHL3 is an actin-binding protein that regulates alpha-actinin-mediated actin bundling: FHL3 localizes to actin stress fibers and enhances cell spreading and stress fiber disassembly. J Biol Chem 278: 24139-24152, 2003.

20. Müller JM, Metzger E, Greschik H, Bosserhoff AK, Mercep L, Buettner R and Schüle R: The transcriptional coactivator FHL2 transmits Rho signals from the cell membrane into the nucleus. EMBO J 21: 736-748, 2002.
21. Martin B, Schneider R, Janetzky S, et al: The LIM-only protein FHL2 interacts with beta-catenin and promotes differentiation of mouse myoblasts. J Cell Biol 159: 113-122, 2002.

22. Labalette C, Renard CA, Neuveut C, Buendia MA and Wei Y: Interaction and functional cooperation between the LIM protein FHL2, CBP/p300, and beta-catenin. Mol Cell Biol 24: 10689-10702, 2004.

23. Shi Y, Chen JY, Yang J, Li B, Chen ZH and Xiao CG: DBC2 gene is silenced by promoter methylation in bladder cancer. Urol Oncol 26: 465-469, 2008.

24. Ding L, Niu C, Zheng Y, et al: FHL1 interacts with estrogen receptors and regulates breast cancer cell growth. J Cell Mol Med (In press).

25. Lin J, Ding L, Jin R, et al: Four and a half LIM domains 1 (FHL1) and receptor interacting protein of $140 \mathrm{kDa}$ (RIP140) interact and cooperate in estrogen signaling. Int J Biochem Cell Biol 41: 1613-1618, 2009.

26. Teng J, Wang ZY, Jarrard DF and Bjorling DE: Roles of estrogen receptor alpha and beta in modulating urothelial cell proliferation. Endocr Relat Cancer 15: 351-364, 2008.

27. Shen SS, Smith CL, Hsieh JT, et al: Expression of estrogen receptors-alpha and -beta in bladder cancer cell lines and human bladder tumor tissue. Cancer 106: 2610-2616, 2006. 